\title{
Endocrine Dysfunction in Neurofibromatosis Type 1 - An Update
}

\author{
Pop Raluca-Monica1,2, Neagoe Radu Mircea ${ }^{3}$, Kolcsar Melinda ${ }^{\star}$, Pașcanu Ionela ${ }^{5}$ \\ ${ }^{1}$ Research Methodology Department, University of Medicine and Pharmacy Tirgu Mures, Romania \\ 2 Endocrinology Outpatient Clinic, Emergency Mureș County Hospital, Tirgu-Mures, Romania \\ 3 Surgery Department, University of Medicine and Pharmacy Tirgu Mures, Tirgu-Mures, Romania \\ 4 Pharmacology and Clinical Pharmacy Department, University of Medicine and Pharmacy Tirgu Mures, Tirgu-Mures, Romania \\ 5 Endocrinology Department, University of Medicine and Pharmacy Tirgu Mures, Romania
}

Background: Neurofibromatosis type 1 is an autosomal dominant disorder associated with multiple neoplasms particularly those of ectodermal origin. Various endocrine pathologies are often present, among them, hyperparathyroidism and follicular thyroid lesion are very rare described and their coincidence in the same patient has not been described in the literature reviewed.

Subject: A 59-years-old woman with clinical manifestation of neurofibromatosis type 1 developed dysphagia, dysphonia, choking sensation. Physical and imagistic examination revealed a multinodular goiter with microfollicular lesion on fine needle aspiration biopsy (FNAB), elevated parathormone levels and severe osteoporosis. The surgically removed thyroid contained a nodule with follicular architecture of uncertain malignant potential; the parathyroid tissue appeared normal.

Discussion and conclusion: This case serves as a reminder to look for non-neurogenic tumors in patients with neurofibromatosis. Clinicians must be aware of the diverse clinical features of this genetic disorder.

Keywords: follicular thyroid lesion, hyperparathyroidism, neurofibromatosis

Received 14 September 2015 / Accepted: 08 January 2016

\section{Introduction}

Neurofibromatosis is an autosomal dominant disorder that affects the bone, the nervous system, soft tissue, and the skin. At least 8 different clinical phenotypes of neurofibromatosis have been identified and are linked to at least 3 genetic disorders [1,2] Despite sharing a common name, these are different phakomatoses both clinically and genetically. The most common form is neurofibromatosis type 1 (NF1) and it is diagnosed based on clinical criteria initially established by the National Institute of Health (NIH) Consensus Development Conference on Neurofibromatosis in 1987. Neurofibromatosis type 2 is differentiated from NF1 by the presence of vestibular schwannomas, juvenile cortical cataract and the missing "café au lait" spots. Schwannomatosis is the third genetic disorder included in the neurofibromatosis' group. Its particular feature is represented by the presence of multiple schwannomas everywhere in the body, except the vestibular nerve [2].

Multiple neoplasms are associated with the features of NF1, particularly those of ectodermal origin - astrocitomas, ependimomas, meningiomas, neurofibrosarcomas, rhabdomiosarcomas, non-lymphocytic leukemia. Neurologic screening is part of the management of patients with neurofibromatosis, considering the fact that clinical signs increase over time.

Various endocrine pathologies are often associated with neurofibromatosis. Short stature and growth hormone de-

* Correspondence to: Melinda Kolcsar

E-mail: melinda.kolcsar@umftgm.ro ficiency are more common in these patients than in general population, although the exact incidence is not known. Precocious puberty, usually caused by a hypothalamic or optic nerve glioma is reported in 3.5\% of the patients. Other endocrine neoplasms related to neurofibromatosis are: pheocromocytoma, hyperparathyroidism, somatostatin producing carcinoid tumors of the duodenal wall, medullary thyroid carcinoma (3). Non-neurogenic tumors are also associated with neurofibromatosis (1).

\section{Case example}

A 59 years-old woman presented in our department with complaints of dysphagia, dysphonia, choking sensation. She was previously diagnosed with NF1, premature ovarian failure and multinodular goiter. Initial physical examination revealed short stature, a dimorphic facies, more than 20 "café au lait" spots, multiple neurofibromas, dispersed all over the body (Fig.1), with axillary and inguinal freckling, pain on percution of the lumbar spine. The thyroid was slightly enlarged, with a $1.5 \mathrm{~cm}$ nodule palpable in the left lobe.

The thyroid ultrasound confirmed the presence of multiple nodules, in both lobes, the largest one of $1.5 \mathrm{~cm}$, in the isthmus, hypoechoic with intense Doppler signal which also had decreased uptake on scintiscan. The FNA cytology report stated a class 4 Bethesda lesion with consequent recommendation of surgical removal of the nodule. Figure $2 \mathrm{a}$ emphasizes the characteristic cytoarchitectural pattern of a follicular neoplasm. It shows several microfollicular cell groups in a clear background (absence 


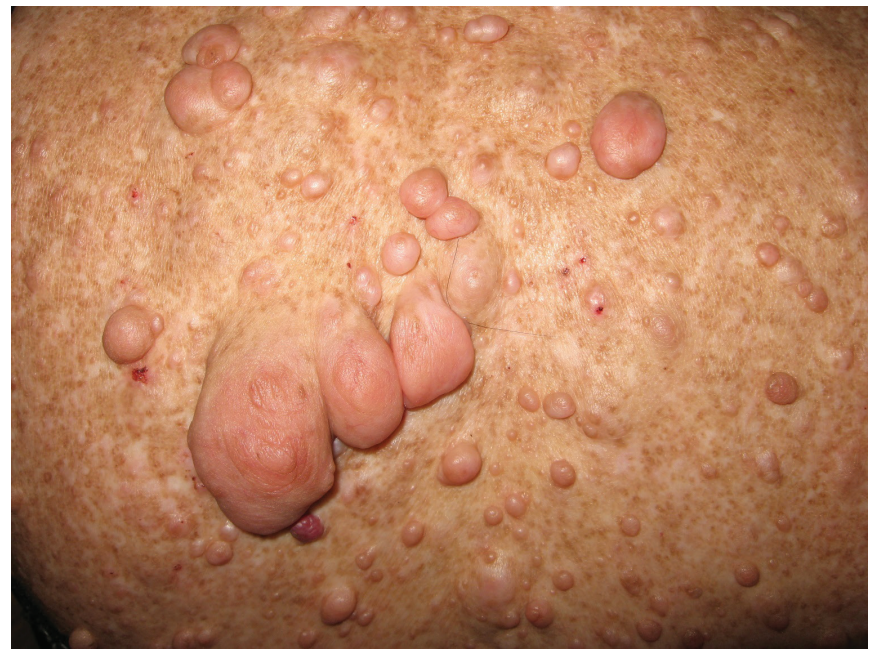

Fig. 1. Neurofibromas in the abdominal region

of colloid). At higher magnification the nuclei of these structures are increased in size, slightly pleomorphic and overlapped but they are round, regular, with a homogeneous chromatin. In some nuclei a centrally placed nucleolus can be visible (Fig. 2b).

Prior to surgery we screened for other known associations of NF 1 with neurological and endocrine disorders and found elevated parathormone values $(182.9 \mathrm{pg} / \mathrm{ml})$,

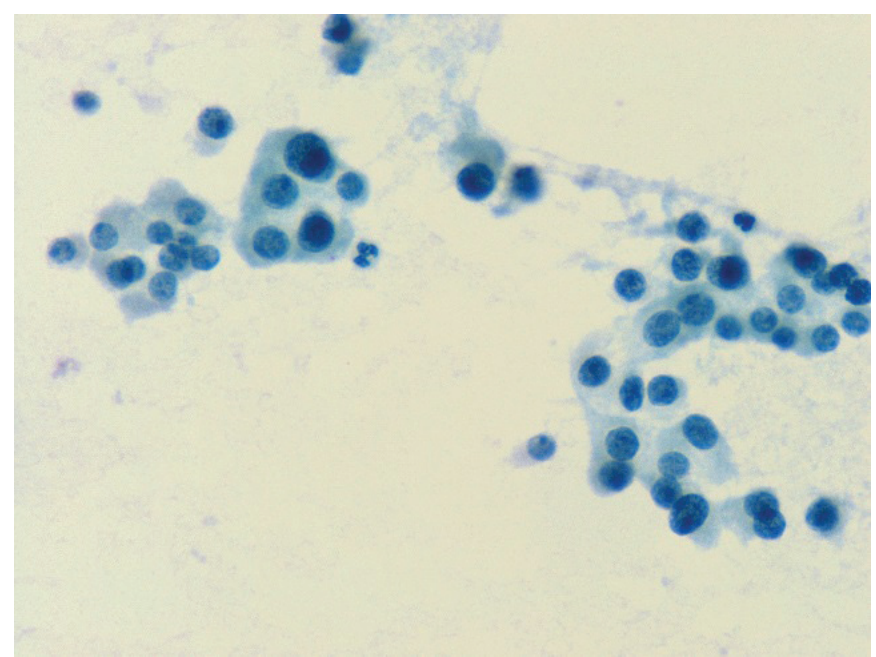

Fig. 2a. The FNA aspect of the follicular neoplasm

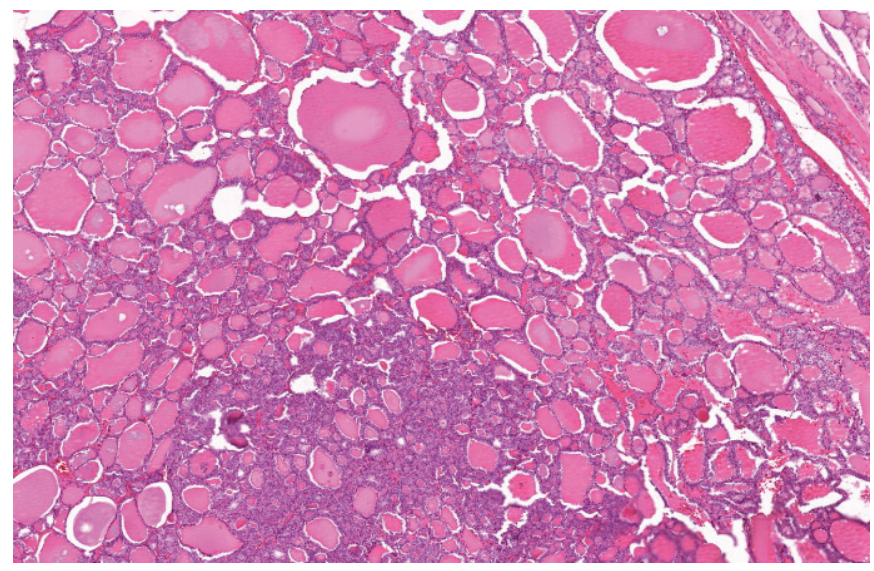

Fig. 3. The follicular pattern of the tumor with normal calcium $(9.17 \mathrm{mg} / \mathrm{dl})$ and low phosphorus levels $(2.39 \mathrm{mg} / \mathrm{dl})$, osteoporosis with low T scores on bone densitometry, consistent with the diagnosis of primary normocalcemic hyperparathyroidism. However, the parathyroid scintiscan did not reveal any abnormal findings. Normal computed tomography imaging has ruled out any cranial manifestation of NF1 and urinary metanephrines levels within normal range excluded the pheochromocytoma.

After the clinical and paraclinical evaluation, total thyroidectomy with subtotal parathyroidectomy was performed. The pathological report showed in a background of chronic lymphocytic thyroiditis, an isthmic tumor with uncertain malignant potential. At low magnification this lesion was encapsulated and had an exclusively follicular pattern of growth with follicles of different sizes, sometimes irregular in shape (Fig. 3). At high magnification, some follicles have round and regular nuclei, with homogenous chromatin and others have nuclear clearing, irregularities of the contours and sometimes rare grooves (Fig. 4). There was no capsular or vascular invasion present.

Post-operatively, the patient developed severe hypocalcemia, which was corrected with calcium infusion. The evolution afterwards was favorable, with no recurrence 5 years after surgery.

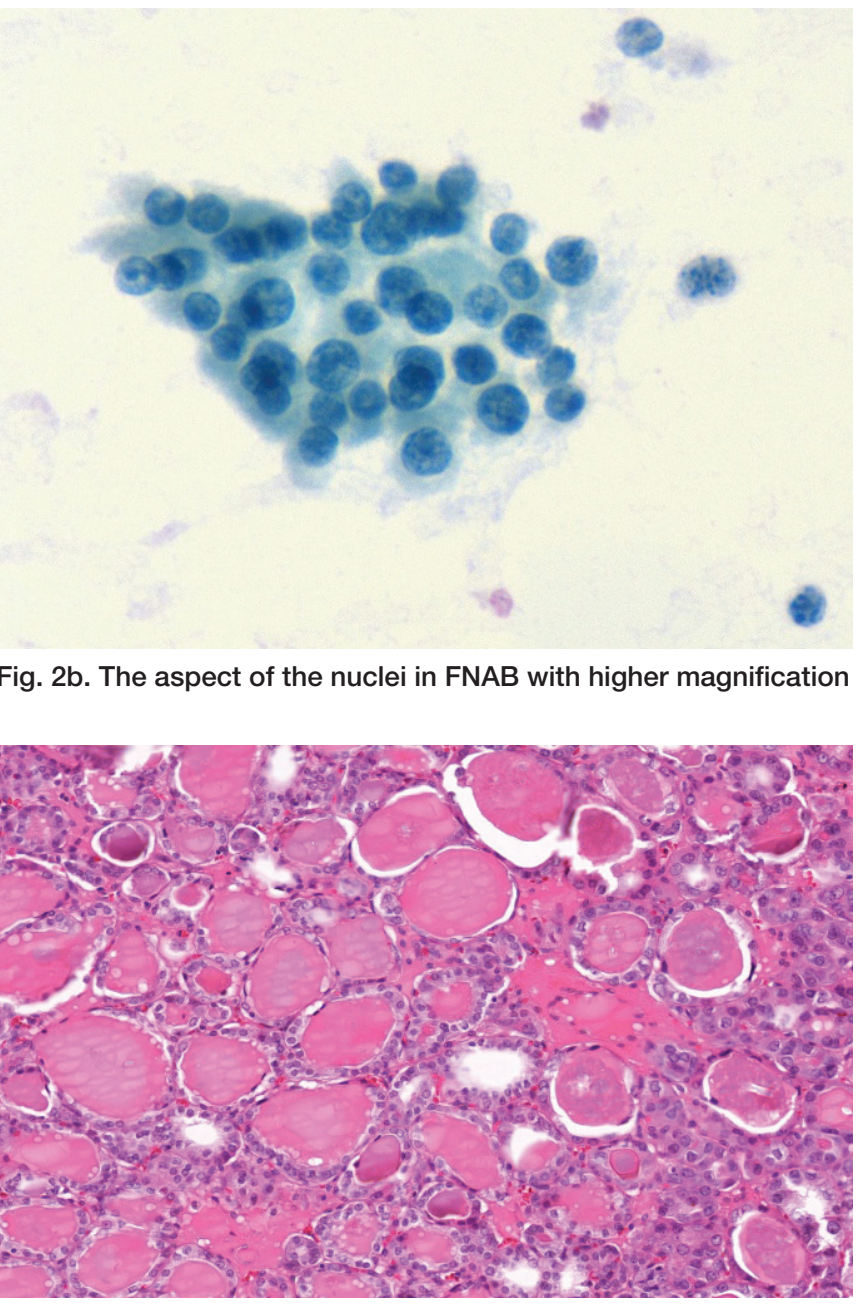

Fig. 4. The aspect of the nuclei with high magnification 


\section{Discussions}

NF1 is one of the most frequent genetic disorders, its prevalence ranging from $1 / 2000$ to $1 / 5000$ in most population based studies [2]. The disease is caused by NF1 gene mutations, located on chromosome $17 \mathrm{q} 11.2$. The protein encoded by this gene, neurofibromin, is tumor suppressor - a negative regulator of the Ras signal transduction pathway. More than 500 different mutations of the NF1 gene have been identified. Most mutations are unique to a particular family. More than $80 \%$ of the germline mutations described in individuals with neurofibromatosis appear to cause severe truncation of the gene product. NF1 is characterized by extreme clinical variability, not only between unrelated individuals but also among affected individuals within a single family. There is no apparent link between the mutations and the clinical manifestations.

The endocrine abnormalities most often associated with neurofibromatosis are precocious puberty, pheocromocytoma, hyperparathyroidism, carcinoid tumors [3]. Specific recommendations for surveillance of patients with NF-1 for other endocrine tumors do not exist. The exact prevalence of hyperparathyroidism in neurofibromatosis patients is not known. There are some reports recommending the screening of all patients with neurofibromatosis for this association [4-10]. The bone lesions described in neurofibromatosis are similar to those found in hyperparathyroidism [11,12]. Our suspicion of hyperparathyroidism, based on the high PTH values with normal calcium, low phosphorus and osteoporosis could not be confirmed by the pathological report, the parathyroid tissue sent for analysis appearing normal. We can conclude that this was a secondary hyperparathyroidism, maybe due to hypovitaminosis $\mathrm{D}$ and osteomalacia $(25-\mathrm{OH}$ vitamin $\mathrm{D}$ value was not available), another disorder described in association with NF 1 [13]. We know that NF1 is one of the most often encountered component of multiple endocrine neoplasias, so, its diagnosis implies the search for other endocrine abnormalities. The association of ectodermal and non-ectodermal derived tumors has been proved, with concomitant features of MEN syndromes and neurofibromatosis present in some cases [14-16], none of which being diagnosed in our case.

There are only a few reports concerning the association of a thyroid lesion with neurofibromatosis [17]. Thyroid malignancies have been described in association with NF1, most often medullary thyroid carcinoma, in some cases associated with pheochromoctoma and primary hyperparathyroidism [14,16].

A follicular lesion of the thyroid is a common diagnosis on FNAB, without the possibility to ascertain its outcome, so surgical treatment is often indicated. The Bethesda classification has improved the management of follicular lesions, although they remain the pitfall of FNAB [18]. In our case the decision for total thyroidectomy was based on the ultrasonography description, the patient's symptoms and the expected difficulty in case of a second intervention due to multiple neurofibromas in the anterior cervical region. Also, the suspicion of primary hyperparathyroidism lead to the choice of surgical treatment. The final pathological description of the nodule was tumor of uncertain malignant potential, without the possibility to ascertain the biologic potential of the lesion. The term "thyroid tumor of uncertain malignant potential" (TT-UMP) is used by pathologists for describing well-differentiated tumors (WDT) showing inconclusive morphological evidence of malignancy or benignity.

The recommendation is that this term should be used only for adequately sampled tumors, when there is real uncertainty about whether the criteria for invasion have been met $[19,20]$. A recent study proposes major and minor criteria for the diagnosis of this type of tumors [21]. Based on these criteria, the patients' tumor is categorized of WDT-UMP. Several studies were performed searching for immunohistochemistry modifications which could be used as markers for these tumors' outcome. None of them showed conclusive results $[22,23]$. It has to be mentioned that DNA microarray gene analysis on thyroid tumors successfully discriminated benign and malignant tumors including borderline lesions [24]. Continuous progress in molecular studies on thyroid tumors hopefully will determine the emergence of a useful diagnostic tool in the future.

This type of tumors are the "grey zone" of thyroid lesions and from the clinical point of view, it complicates the future management of our patient. There is no treatment protocol or a consensus regarding the post-operative follow-up for this type of lesion. Although radioiodine treatment is not indicated, the potential for metastases or recurrence is not known and careful follow-up is recommended [20]. There is no express recommendation for suppressive doses of levothyroxine or a clear timeframe for ultrasound studies. Our opinion would be to administer adequate doses of levothyroxine in order to keep the TSH level in the lower normal range and to perform at least annual checkups, including ultrasound and, maybe, thyroglobulin level. In order to better classify and treat this type of tumors, further studies are needed, regarding the survival rate or the metastases percentage.

In summary, we described the case of a 59 years old woman with neurofibromatosis who associated a thyroid tumor of uncertain malignant potential and secondary hyperparathyroidism. Although this association can be simply a coincidence, prompt diagnosis is necessary in order to provide optimum care for our patients with neurofibromatosis type 1 .

\section{Conclusions}

The association of thyroid lesions should be sought in patients with clinical features of neurofibromatosis and an ultrasonography of the thyroid gland should be performed. Although hyperparathyroidism caused by an adenoma is described in patients with NF1, other causes of elevated 
PTH values should be sought also. Clinicians must be aware of the diverse clinical features of this genetic disorder.

\section{Acknowledgement}

We would like to thank Professor Angela Borda from the Histology Department of the University of Medicine and Pharmacy Tirgu Mures for the pathology images and their interpretation.

\section{References:}

1. Hirbe AC, Gutmann DH. Neurofibromatosis type 1: A multidisciplinary approach to care. The Lancet. Neurology. 2014;13(8):834-843.

2. Rasmussen JM, Friedman S. NF1 gene and neurofibromatosis 1. American Journal of Epidemiology. 2000;151:33-40.

3. S Melmed, K Polonsky, P Larsen, $\mathrm{H}$ Kronenberg. Williams textbook of endocrinology: 12th ed. Philadelphia, Saunders Elsevier 2012;555-567.

4. Oates E, Bankoff MS, Vogelzang PJ. Parathyroid adenoma associated with neurofibromatosis: correlative scintigraphic and magnetic resonance imaging. Clin Nucl Med. 1989;14:168-70.

5. Bijou F, Vargas F, Valentino R, et al. Association of type 1 neurofibromatosis and primary hyperparathyroidism. Presse Med. 2002;31:1604-5.

6. lihara M, Okamoto T, Obara T et al. Water-clear cell parathyroid adenoma causing primary hyperparathyroidism in a patient with neurofibromatosis type 1: report of a case. Surg Today. 2007;37:884-7.

7. Collicott PE, Stivrins TJ, Hoppe LB. Von Recklinghausen's neurofibromatosis and primary hyperparathyroidism: a case report and literature review. Nebraska Medical Journal. 1986;1:435-7.

8. Kalkan E, Waguespack SG. Endocrine tumors associated with neurofibromatosis type 1, peutz-jeghers syndrome and other familial neoplasia syndromes. Frontiers of Hormone Research. 2013;41:166181.

9. Heervae E, Leinonen P, Kuorilehto T et al. Neurofibromatosis 1-Related Osteopenia Often Progresses to Osteoporosis in 12 Years. Calcified Tissue International. 2013:92:23-27.

10. Al-Wahhabi B. Parathyroid adenoma and bilateral pheochromocytoma in a patient with neurofibromatosis. Ann Saudi Med. 2005;25:255-257.
11. Sabatini C, Milani D, Menni F, Tadini G, Esposito S. Treatment of neurofibromatosis type 1. Current treatment options in neurology. 2015;17(6):355-360

12. Gurleyik F, Ocak C, Bahadir D. Neurofirbomatosis type 1 and primary hyperparathyroidism with spinal deformity and osteoporosis. Acta Chir Belg. 2009;109:123-5.

13. Gkaliagkousi E, Erlic Z, Petidis K et al. Neurofibromatosis type 1: should we screen for other genetic syndromes? A case report of co-existence with multiple endocrine neoplasia 2A. Eur J Clin Invest. 2009;39:828832.

14. Diliberti JH, Andrews AM, Buist $\mathrm{N}$ et al. Myotonic dystrophy and hyperparathyroidism: association with neurofibromatosis and multiple endocrine adenomatosis type 2A. J Neurol Neurosurg Psychiatry. 1988;51:1578-1580.

15. Madson Q, Stratakis CA. Solid tumors associated with multiple endocrine neoplasias. Cancer genetics and cytogenetics. 2010;203:30-36.

16. TorunerF, CimenAR, Karakoc A et al. The association of neurofibromatosis, bilateral pheochromocytoma and primary hyperparathyroidism. Exp Clin Endocrinol Diabetes. 2007;115:48-70.

17. De Lellis RA, Lloyd RV, Heitz PU. Pathology and genetics: tumours and endocrine organs. 3rd ed. Geneva: WHO, 2004

18. Edmund S, Cibas SZ. The Bethesda System for Reporting Thyroid Cytopathology. Am J Clin Pathol. 2009;13:658-665.

19. Rosai J, LiVolsi VA, Sobrinho-Simoes $M$ et al. Renaming papillary microcarcinoma of the thyroid gland: the Porto proposal. Int J Surg Pathol. 2003;11:249-251.

20. Véronique Hofman, Sandra Lassalle, Christelle Bonnetaud et al. Thyroid tumours of uncertain malignant potential: frequency and diagnostic reproducibility. Virchows Arch. 2009;455(1):21-33.

21. Fatma El-Zahraa, Salah El-Deen Yassin. Diagnostic criteria of well differentiated thyroid tumor of uncertain malignant potential; a histomorphological and immunohistochemical appraisal. Journal of the Egyptian National Cancer Institute. 2015;27(2):59-67.

22. Nechifor-Boila A, Borda A, Sassolas G et al. Thyroid tumors of uncertain malignant potential: morphologic and imunohistochemical analysis of 29 cases. Pathol Res Pract. 2015;211(4):320-5.

23. Arora N, Scognamiglio T, Lubitz CC et al. Identification of borderline thyroid tumors by gene expression array analysis. Cancer. 2009;115:5421-31.

24. Baloch ZW, LiVolsi VA. Our approach to follicular-patterned lesions of the thyroid. J Clin Pathol. 2007;60:244-50. 